\title{
Un apunte de Derecho privado sobre las cooperativas en el entorno agroalimentario
}

\section{Las entidades cooperativas}

\subsection{Ideas introductorias con apoyo del Derecho privado}

La Constitución española contiene una mención expresa sobre las entidades cooperativas ${ }^{1}$, señalando que serán los poderes públicos los que han de fomentar las entidades cooperativas a través de una adecuada regulación. Referencia genérica que sólo establece la necesidad de que las autoridades estatales impulsen las entidades cooperativas mediante la aprobación de la correspondiente normativa. Así, la delimitación conceptual de las sociedades cooperativas hay que concretarla en razón de diversos elementos de naturaleza jurídica, económica e ideológica.

La definición contenida en la norma la precisa como la asociación de personas unidas en régimen de libre adhesión y baja voluntaria que tiene por finalidad llevar a cabo actividades empresariales para satisfacer las propias necesidades de sus integrantes y sus aspiraciones económicas y sociales ${ }^{2}$.

* Universidad de Jaén, España.

${ }^{1}$ Art. 129, apartado $2^{\circ}$ de la Constitución española. Para ampliar esta información puede consultarse F. Vicent Chuliá, Capitulo I. Introducción, en: J.I. Peinado Gracia (dir.), T. Vázquez Ruano (coord.), Tratado de Derecho de Sociedades Cooperativas, Valencia 2019, pp. 69-74.

${ }^{2}$ Art. 1 de la Ley 27/1999, de 16 de julio, de Cooperativas (BOE No. 170, de 17 de julio. En adelante, LC) y la Ley 5/2011, de 29 de marzo, de Economía Social (BOE No. 76, de 30 de marzo. En adelante, LES). La Ley de Cooperativas nacional va a resultar de aplicación directa 
Y que, a su vez, dispone de una estructura organizativa y un funcionamiento democrático en consonancia con la observancia de los valores y principios cooperativos reformulados por la Alianza Cooperativa Internacional (en adelante, ACI). De este modo, la cooperativa va a explotar su objeto social para satisfacer directamente las necesidades de sus socios y no obteniendo un beneficio social repartible en proporción a las aportaciones al capital social, sino que los socios serán reintegrados cuando se hubiera establecido en los Estatutos sociales en razón del límite que establece la Ley y de acuerdo con la distribución parcial del excedente realizado con las entregas o prestaciones que los socios hacen a la propia entidad. Las cooperativas son entidades mercantiles de carácter especial titulares de una empresa en la que los socios se singularizan por tener ánimo de lucro y en la que los beneficios sociales se distribuyen entre los mismos por retornos o por precios $^{3}$. Es una agrupación

en los supuestos que de modo expreso se recogen en sus disposiciones, a saber: las sociedades cooperativas que desarrollen su actividad cooperativizada en el territorio de varias Comunidades autónomas (excepto cuando en una de ellas se desarrolle con carácter principal); y a las sociedades cooperativas que realicen principalmente su actividad cooperativizada en Ceuta y Melilla. Además, la Ley de Cooperativas nacional resulta de aplicación directa en aquellos aspectos que son de competencia estatal exclusiva, como los concursales, las cuestiones procesales o los contables.

Las normas autonómicas vigentes al momento de elaborar este trabajo son las que se relacionan a continuación: la Ley 14/2011, de 23 de diciembre, de Sociedades Cooperativas Andaluzas y el Decreto 123/2014, de 2 de septiembre, por el que se aprueba el Reglamento de la Ley 14/2011, de 23 de diciembre, de Sociedades Cooperativas Andaluzas; Decreto Legislativo 2/2014, de 29 de agosto, del Gobierno de Aragón, por el que se aprueba el texto refundido de la Ley de Cooperativas de Aragón; la Ley 6/2013, de 6 de noviembre, de Cooperativas de Cantabria; la Ley 4/2002, de 11 de abril, de Cooperativas de la Comunidad de Castilla y León; la Ley 11/2010, de 4 de noviembre, de Cooperativas de Castilla-La Mancha (modificada por la Ley 4/2017, de 30 de noviembre, de Microempresas Cooperativas y Cooperativas Rurales de Castilla-La Mancha); la Ley 12/2015, de 9 de julio, de Cooperativas de Cataluña; la Ley 2/1998, de 26 de marzo, de Sociedades Cooperativas de Extremadura; la Ley 5/1998, de 18 de diciembre, de Cooperativas de Galicia; la Ley 8/2006, de 16 de noviembre, de Sociedades Cooperativas, de la Región de Murcia; la Ley 4/2001, de 2 de julio, de Cooperativas de La Rioja; la Ley 1/2003 de 20 de marzo, de cooperativas de las Islas Baleares; la Ley 4/1999, de 30 de marzo, de Cooperativas de la Comunidad de Madrid; la Ley 4/2010, de 29 de junio, de Cooperativas del Principado de Asturias; Decreto Legislativo 2/2015, de 15 de mayo, del Consell, por el que aprueba el Texto Refundido de la Ley de cooperativas de la Comunidad Valenciana; la Ley Foral 14/2006, de 11 de diciembre, de Cooperativas de Navarra. A las que se añaden, la Ley 4/1993, de 24 de junio, de Cooperativas de Euskadi (modificada por la Ley 8/2006, de 1 de diciembre y por la Ley 6/2008, de 25 de junio, de la Sociedad Cooperativa Pequeña de Euskadi) y el Anteproyecto de Ley de Cooperativas de Euskadi; el Proyecto de Ley de Cooperativas de Canarias de 2018.

3 A pesar de la extendida calificación doctrinal de las cooperativas como sociedades mercantiles, esta modalidad societaria no se ha reflejado de forma unitaria en la legislación española ni en la jurisprudencia de nuestro Tribunal Constitucional. La negación de la naturaleza mercantil de estas sociedades implica que no se encuadren entre las materias que la Constitución española reserva a la competencia exclusiva del Estado (art. $149.1^{\circ}$ de la Constitución española), sino que 
voluntaria que se distingue porque su organización corporativa y financiera respeta los principios de la ACI. La Ley de Cooperativas española define la sociedad cooperativa del siguiente modo 4 : "[...] sociedad constituida por personas que se asocian, en régimen de libre adhesión y baja voluntaria, para la realización de actividades empresariales, encaminadas a satisfacer sus necesidades y aspiraciones económicas y sociales, con estructura y funcionamiento democrático [...] y se regirá [...] conforme a los principios formulados por la Alianza Cooperativa Internacional, en los términos resultantes de la presente Ley $[\ldots] "$..

Por tanto, cabe considerar que las entidades cooperativas son agrupaciones voluntarias y democráticas de personas que tienen una misma necesidad económica y social y, a fin de satisfacerla, crean una empresa que explotan en común, bajo el principio de la ayuda mutua, desarrollando una economía de servicio.

La distinción de las sociedades cooperativas en base al nivel de integración lleva a distinguir las cooperativas de primer grado ${ }^{5} \mathrm{y}$ las cooperativas de segundo (o ulterior) grado que son las que cuentan entre sus socios con, al menos, dos cooperativas de base, aunque también pueden integrar como socios a otras personas jurídicas, públicas o privadas, y empresarios individuales $^{6}$. El objeto de las cooperativas de segundo grado va a ser promover, coordinar y desarrollar fines económicos comunes de sus socios, y reforzar e integrar la actividad económica de los mismos. Razón que ha sido determinante respecto del incremento de las cooperativas agrarias. En cuanto a su funcionamiento, la representación de cada persona jurídica-socio (co-

-por el contrario-se trate de una materia cuya competencia legislativa se reconoce a las Comunidades autónomas en base a lo previsto en sus propios Estatutos de autonomía (art. 148 de la Constitución española). J.I. Peinado Gracia, Capítulo I. Normas y ámbito de aplicación, en: J.I. Peinado Gracia (dir.), T. Vázquez Ruano (coord.), Tratado de Derecho..., pp. 125-128; F. Vicent Chuliá, Capítulo I..., pp. 99-105. Razón que ha traído como consecuencia que, a nivel interno, los Estatutos de autonomía de las Comunidades autónomas de España hayan reclamado la competencia legislativa en materia cooperativa y que -junto a la norma estatal- existan normas cooperativas autonómicas.

${ }^{4}$ Art. 1 de la LC. Véanse Mª.J. Morillas Jarillo, Capitulo II. Concepto y clases de cooperativas, en: J.I. Peinado Gracia (dir.), T. Vázquez Ruano (coord.), Tratado de Derecho..., pp. 163-170; F. Vicent Chuliá, Capitulo I..., pp. 90-98.

${ }^{5}$ Art. $1.1^{\circ}$ de la LC.

${ }^{6}$ Art. 77 apartado 1 de la LC. Para ampliar esta consideración, pueden consultarse R. Alfonso Sánchez, La integración cooperativa y sus técnicas de realización: la cooperativa de segundo grado, Valencia 2000, pp. 361-377; C. Vargas Vasserot, E. Gadea Soler, F. Sacristán Bergia, Régimen económico, integración, modificaciones estructurales y disolución, Madrid 2017, pp. 211-215; J.M. Vázquez Pena, Capitulo XI. II. Formas personificadas de integración: cooperativas de segundo grado, en: J.I. Peinado Gracia (dir.), T. Vázquez Ruano (coord.), Tratado de Derecho..., pp. 1218-1227. 
operativa) en la Asamblea general va a corresponder a la persona que tenga su representación legal. Como norma habitual, y si así lo prevén los Estatutos sociales, en las cooperativas de segundo grado el voto de los socios podrá ser proporcional a su participación en la actividad cooperativizada y/o al número de socios activos que integran la cooperativa asociada. Aunque con la limitación máxima de que ningún socio puede ostentar más de un tercio de los votos totales, salvo que la sociedad esté integrada sólo por tres socios (pudiendo ostentar el 40\%); y si la integrasen dos socios, los acuerdos deberán adoptarse por unanimidad. La cuantificación de los votos de las entidades que no ostenten la condición de cooperativas no podrá alcanzar el $40 \%$ de los votos sociales. El haber líquido resultante se distribuye entre las socios en proporción al importe del retorno percibido en los últimos cinco años, o desde la constitución de la entidad secundaria si no se alcanza dicho plazo; o al volumen de actividad cooperativizada desarrollada por cada socio con la cooperativa durante el citado período; o al número de miembros de cada entidad agrupada. La norma establece, asimismo, la posibilidad de que las sociedades cooperativas de segundo grado se transformen en cooperativas de primer grado, lo que supone que sean absorbidas las cooperativas-socios.

Pero, además, las sociedades cooperativas se clasifican según el criterio del objeto de la entidad o la actividad mutualizada. En cuyo caso, la norma determina un número indicativo de tipos de cooperativas de primer grado y su regulación específica ${ }^{7}$. De las previstas en la norma, conviene hacer referencia somera a: las cooperativas de consumidores y usuarios en las que se mutualiza la necesidad de consumo de bienes y servicios de sus socios; las de viviendas en las que se mutualiza la necesidad de alojamiento9; las de crédito en las que se mutualiza el uso de servicios financieros activos y pasivos $^{10}$; las de trabajo asociado en relación con la capacidad laboral para cualquier labor o industria ${ }^{11}$; las sanitarias que mutualizan el ejercicio de actividades sanitarias y el uso de las mismas ${ }^{12}$; y las de enseñanza respecto del ejercicio de actividades docentes y su uso ${ }^{13}$. Por su parte, y sin sustituir a sus socios en el ejercicio principal de la actividad, cabe referenciar las cooperativas de servicios en las que se mutualizan las necesidades derivadas

${ }^{7}$ Art. 6 de la LC. Para más información vea Ma . J. Morillas Jarillo, Capítulo II..., pp. 173-180. Y un mayor estudio de cada modalidad cooperativa se hace en: J.I. Peinado Gracia (dir.), T. Vázquez Ruano (coord.), Tratado de Derecho..., vol. II.

8 Art. 88 de la LC.

${ }_{9}$ Arts. 89 a 92 de la LC.

${ }^{10}$ Art. 104 de la LC.

${ }^{11}$ Arts. 80 a 87 de la LC.

${ }_{12}$ Art. 102 de la LC.

${ }^{13}$ Art. 103 de la LC. 
de actividades industriales, de servicios, profesionales o artísticas ${ }^{14}$; las de la mar que mutualizan las necesidades derivadas de actividades pesqueras ${ }^{15}$; las de transportistas en las que se mutualizan las necesidades derivadas de actividades de transporte ${ }^{16}$; y las de seguros que mutualizan el ejercicio de la actividad aseguradora en aquellos ámbitos en los que se encuentren legalmente habilitadas ${ }^{17}$. Por último, las cooperativas agrarias en las que se mutualizan las necesidades de uso o consumo de bienes y servicios derivadas de una actividad económica agraria, ganadera o forestal sin sustituir a sus socios en el ejercicio principal de la actividad ${ }^{18}$; y las cooperativas de explotación comunitaria de la tierra en las que se mutualiza el ejercicio de la labor agraria ${ }^{19}$.

Junto a estas modalidades cooperativas, cabe distinguir las cooperativas integrales que se distinguen porque su objeto social es plural ${ }^{20}$; las cooperativas mixtas que cuentan con la doble naturaleza de cooperativas y de entidades de capital en ciertos aspectos; y las cooperativas sin ánimo de lucro en las que se gestionan servicios de interés general ${ }^{21}$. En estas últimas, quedan encuadradas las cooperativas de iniciativa social que desempeñan labores asistenciales o se dedican a la integración laboral de personas con cualquier causa de exclusión social, o satisfacen necesidades sociales no atendidas por el mercado ${ }^{22}$.

Asimismo, se reconoce una clasificación que se corresponde con la dimensión de la cooperativa en relación con el número de socios, tal es el caso de las cooperativas de menor tamaño como las de trabajo asociado y las de explotación comunitaria de la tierra. Respecto de las que se establecen ciertas especialidades en relación con su constitución, inscripción y funcionamiento $\mathrm{y}$, en igual sentido, en lo que hace a sus órganos sociales y al régimen económico que le es propio. Y, además, ha de tenerse en cuenta que la integración empresarial de las sociedades cooperativas desde la perspectiva institucional puede hacerse a través de la creación de grupos cooperativos ${ }^{23}$, los cuales

${ }^{14}$ Art. 98 de la LC.
${ }^{15}$ Art. 99 de la LC.
${ }^{16}$ Art. 100 de la LC.
${ }_{17}$ Art. 101 de la LC.
${ }^{18}$ Art. 93 de la LC.
${ }^{19}$ Arts. 94 a 97 de la LC.
${ }^{20}$ Art. 105 de la LC.
${ }_{22}^{22}$ Aisposición Adicional $1^{\text {a }}$ y $9^{\text {a }}$ de la LC.
${ }^{23}$ Véase el art. 78 de la LC. Sobre esta materia, pueden consultarse: J.M Embid Irujo, R. Alfonso Sánchez, Capitulo XI. III. Formas no personificadas de integración: grupos cooperativos, en: J.I. Peinado Gracia (dir.), T. Vázquez Ruano (coord.), Tratado de Derecho..., 
se determinan como un modo de agrupación sin vinculación patrimonial. Y ello ha de considerarse de forma positiva para el desarrollo de las pequeñas y medianas empresas. El grupo cooperativo consiste en el acuerdo por el que dos o más cooperativas deciden ceder a una entidad cabeza de grupo la posibilidad de que ejercite facultades o emita instrucciones de obligado cumplimiento para las demás. Por lo que se produce una unidad de decisión en el ámbito de dichas facultades y de las competencias que le son atribuidas; y teniendo en cuenta que la emisión de instrucciones podrá afectar a distintos ámbitos de gestión, administración o gobierno. Ya que se establecen relaciones asociativas entre las cooperativas que hacen que actúen con una unidad de decisión propia. Por ende, la subordinación no se establece a favor de una sociedad independiente, sino en lo que hace a una entidad en la que participa un conjunto de entidades dominadas. Es decir, las entidades ceden el control a una estructura común en la que todas participan.

\subsection{Especialidad de las cooperativas agroalimentarias}

En el sector agroalimentario la conceptualización de la cooperativa agroalimentaria trae causa en los criterios subjetivo y objetivo que le son propios. Pues se trata de una cooperativa integrada por agricultores y/o ganaderos que ponen en común sus recursos en determinadas actividades y sus servicios, a fin de poder comercializar en conjunto los productos. De este modo las cooperativas agroalimentarias van a asociar a personas que son titulares de explotaciones agrícolas, ganaderas o forestales, cuya finalidad prioritaria va a ser la realización de actividades y operaciones para mejorar el aprovechamiento de estas explotaciones propias, de sus elementos o componentes de la cooperativa y para la mejora de la población agraria y del desarrollo del mundo rural $^{24}$. La actuación de estas cooperativas destaca en el entorno territorial y social en el que están ubicadas. También han de incluirse entre los socios de dichas entidades a las sociedades agrarias de transformación, las comunidades de regantes, las de aguas, las de bienes y las sociedades civiles o mercantiles que tengan el mismo objeto social o actividad complementaria.

pp. 1229-1260; R. Server Izquierdo, E. Meliá Martí, Caracterización empresarial de los grupos y otras formas de integración cooperativa al amparo del nuevo marco legislativo, „Revista de estudios cooperativos" 1999, No. 69, pp. 9-41; C. Vargas Vasserot, E. Gadea Soler, F. Sacristán Bergia, Régimen económico..., pp. 211-215; C. Vargas Vasserot, Integración y diferenciación cooperativa: de las secciones a los grupos de sociedades, "Boletín de la Asociación Internacional de Derecho Cooperativo" 2010, No. 44, pp. 159-176.

${ }^{24}$ Art. 93 de la LC. 
Las cooperativas agroalimentarias de primer grado tienen por finalidad la producción de alimentos y las de segundo (o ulterior) grado se ocupan de la comercialización de los productos agrícolas de aquéllas (de primer grado). Fundamentalmente, en el sector de las frutas y hortalizas, los aceites de oliva, vinos, lácteos, ovino y caprino, cereales y la alimentación animal. Asimismo, y junto a éstas, se crean cooperativas de suministros para proporcionar a sus integrantes inputs necesarios para la producción del producto; cooperativas de servicios de comercialización cuya finalidad es la transformación, distribución y promoción de los propios productos agrícolas; y las cooperativas de crédito empleadas como instrumento de obtención de financiación o a fin de cubrir las necesidades financieras de sus socios y de terceros.

Por su parte, las referidas cooperativas de explotación comunitaria de la tierra o de producción agrícola, asocian a los titulares de derechos de uso y aprovechamiento de tierras o inmuebles susceptibles de explotación agraria y que van a ceder esos derechos a la cooperativa, prestando o no su trabajo a la misma. En igual sentido, pueden asociar a personas físicas que, sin ceder a la cooperativa los derechos indicados, le prestan su trabajo para la explotación en común de los bienes cedidos por los socios y del resto que posea la cooperativa ${ }^{25}$.

En el ámbito interno español conviene aludir a la entidad Cooperativas Agroalimentarias de España, órgano en el que están representadas las Federaciones Regionales de cooperativas agroalimentarias ${ }^{26}$, lo que supone la asociación del $80 \%$ (aproximadamente) de las cooperativas agroalimentarias nacionales (alrededor de 1.172.226 socios cooperativistas). La entidad Cooperativas Agroalimentarias representa a unas 4.000 cooperativas, siendo casi el 13\% de las mismas de la industria agroalimentaria y uniendo a más de un millón de productores. Los objetivos principales de esta Organización son: de un lado, la tutela de los intereses económicos y sociales del sector cooperativo agroalimentario frente a la Administración General del Estado y a las instituciones de la UE y, a su vez, ante las organizaciones estatales que representan los agentes socioeconómicos de los sectores en los que llevan a cabo su actividad las cooperativas agroalimentarias. De otro, la promoción y desarrollo del cooperativismo agroalimentario en el momento vigente y en razón de los retos próximos o de fututo del sector agroalimentario. La

${ }_{25}$ Art. 94 de la LC.

${ }^{26}$ Esta Institución se creó en 1990 como "Confederación de Cooperativas Agrarias de España (CCAE)". Actualmente, Cooperativas Agroalimentarias de España es la organización que representa y defiende los intereses tanto económicos, como de carácter social del movimiento cooperativo agrario a nivel interno. Cooperativas agro-alimentarias, http://www.agro-alimentarias. coop/inicio [consultado: 11 de octubre de 2021]. 
tendencia general de las cooperativas, desde el 2007, ha sido positiva, incrementando su facturación $(+46 \%)$ y el empleo $(+22 \%)$ produciéndose una cierta concentración empresarial del colectivo. Por un lado, ha disminuido un $8 \%$ el número de cooperativas $\mathrm{y}$, por otro, ha aumentado su facturación un $40 \%$, lo que pone de manifiesto los esfuerzos realizados por captar un mayor valor añadido. Asimismo, las cooperativas han incrementado de manera significativa su actividad exportadora, lo que viene a reforzar la importancia de la integración comercial para alcanzar la dimensión necesaria para competir en los mercados exteriores. La facturación de las cooperativas en los mercados exteriores representa el $31,5 \%$ de la facturación total del colectivo y el $31 \%$ de las cooperativas españolas realiza actividad exportadora. Las cooperativas representan un $16 \%$ de la facturación exportadora del conjunto del sector agroalimentario español (sin pesca). En definitiva, las cifras lo que reflejan es la importancia del cooperativismo y su papel en el sector agroalimentario español, que mantienen el crecimiento económico y conservan su compromiso de fidelidad con las zonas rurales donde se asientan ${ }^{27}$.

\section{Anotaciones sobre los principios de la Alianza Cooperativa Internacional}

La estructura organizativa y el funcionamiento de la sociedad cooperativa, como se ha indicado, sigue el sistema democrático en consonancia con la observancia de unos valores y principios concretos, los Principios cooperativos reformulados por la ACI en el Congreso de Manchester de $1995^{28}$. La ACI en la Declaración sobre la Identidad Cooperativa estableció una delimitación del concepto tendente a homogeneizar los criterios vigentes en el ámbito cooperativo internacional y lo concretó de la siguiente forma, se trata de: una asociación autónoma de personas que se han unido de forma voluntaria para satisfacer sus necesidades y aspiraciones económicas, sociales y culturales en común, mediante una empresa de propiedad conjunta $y$ de gestión democrática.

Esta Declaración completa la definición de la entidad cooperativa con un conjunto de Valores cooperativos como normas éticas de actuación (Dec-

27 Puede consultarse el espacio electrónico siguiente: https://www.agro-alimentarias.coop/ cooperativismo_en_cifras [consultado: 13 de octubre de 2021].

28 Alianza Cooperativa Internacional, XXXI Congreso y Asamblea General de la ACI (Manchester, septiembre de 1995) en el que se adoptó una Declaración sobre la Identidad Cooperativa https://www.ica.coop/es [consultado: 13 de octubre de 2021]. 
laración sobre los Valores), cuáles son ${ }^{29}$ : la autoayuda respecto de la acción conjunta y la responsabilidad mutua (el desarrollo individual pleno se logra con la asociación con el resto, acción conjunta y responsabilidad mutua), y de cuyo valor trae causa la solidaridad, entendida como el predominio del interés general o colectivo y el trato justo respecto de los que integran la cooperativa (socios, no socios aunque con vinculación a la entidad y empleados) y, a su vez, entre cooperativas; la autorresponsabilidad que asumen los socios en cuanto a la entidad cooperativa y se ocupan de su promoción y difusión; la democracia aplicada a su gestión y respecto de su funcionamiento; la igualdad del socio como unidad fundamental de la cooperativa; la equidad del tratamiento de los socios cooperativos y en la contribución de ellos en la cooperativa; la honestidad en relación con el desempeño de prácticas éticas y acordes con los buenos usos; la transparencia en la información sobre sus operaciones; y la vocación social.

Los valores referidos se ejecutan a través de los Principios cooperativos, los cuales se concretan en los que siguen. El principio de adhesión voluntaria y abierta, en la medida en que formar parte de la organización cooperativa depende de la libre voluntad y decisión individual y, además, en cuanto a que la entidad va a estar abierta a todas las personas capaces de utilizar sus servicios y dispuestas a aceptar las responsabilidades de ser socio, sin discriminación social, política, religiosa, racial o de sexo, en razón del reconocimiento de la dignidad personal. Sin perjuicio, de posibles límites respecto a quiénes pueden ser socios en función de la finalidad específica que caracterice a la sociedad cooperativa. El principio de gestión democrática por parte de los socios en la organización, lo que implica la participación activa de los socios en la adopción de las decisiones y en la política de la entidad, tanto si la cooperativa es de primer grado (un socio, un voto), como en las de segundo o ulterior grado. Asumiendo la correspondiente responsabilidad de la gestión a favor del interés social frente al particular de los socios. El principio de participación económica de los socios, pues los sujetos que integran la cooperativa van a contribuir de forma equitativa al capital de la misma y lo han de gestionar democráticamente. Esto es, que parte de dicho capital es propiedad común de la cooperativa y, pese a que el capital es un elemento preciso, no puede entenderse que sea esencial. Si bien, en el caso de que proceda, los socios pueden recibir una compensación que se halla limitada en razón del capital entregado al momento de formar parte de la sociedad. La autonomía e independencia como principios de gestión de la sociedad cooperativa por parte de los socios, primando la organización

29 Vid. M. J. Morillas Jarillo, Capitulo II..., pp. 163-170. 
democrática y manteniendo la autonomía de la entidad cooperativa en la libre adopción de sus decisiones y sus relaciones con otras organizaciones, incluido el Gobierno estatal. Por consiguiente, si llegan a acuerdos con organizaciones externas, incluyendo los gobiernos, o aumentan su capital de fuentes externas, lo harán de forma que aseguren el control democrático de sus miembros y manteniendo la autonomía de la cooperativa. El principio de educación, formación e información a los socios que integran las sociedades cooperativas, así como a sus representantes, directivos y empleados. Del mismo modo, la formación e información se ha de proporcionar también desde una perspectiva común, es decir, al público en general. Y, por último, el principio de cooperación entre cooperativas y el principio del interés por la comunidad. Lo que significa que la entidad cooperativa sirve a sus socios de un modo eficaz y fortaleciendo el movimiento cooperativo, trabajando de manera conjunta mediante estructuras locales, regionales, nacionales e internacionales. Junto a las necesidades de los socios, la cooperativa ha de actuar con responsabilidad en la adopción de las decisiones propias de la misma y, en concreto, en cuanto al lugar en el que se ubica y en lo que se refiere a la promoción de la participación en el desarrollo de la comunidad. Las cooperativas trabajan para el desarrollo sostenible de sus comunidades, en interés de la comunidad a través de políticas aprobadas por sus miembros.

En definitiva, la finalidad de las sociedades cooperativas en el ámbito agrario es tratar de alcanzar las necesidades y aspiraciones que son comunes a sus socios mediante la empresa, fundamentalmente de carácter económico y social. Y ello determina el objeto de la entidad o la actividad cooperativizada que la singulariza.

\section{El funcionamiento y gobierno de una entidad cooperativa}

El régimen de autogestión por parte de los que ostentan la condición de socio es singular de las sociedades cooperativas, en la medida en que los socios no sólo participan económicamente realizando la actividad cooperativizada, sino también se van a encargar de la gestión de la sociedad. La regulación de los órganos sociales en las cooperativas se basa en un modelo de participación orgánica de los socios que es propio de las entidades de base mutualista. Los órganos sociales necesarios de una cooperativa son la Asamblea general que reúne a todos los socios y es el órgano máximo de decisión, pues sus decisiones vinculan a la totalidad de los que la conforman ${ }^{30}$; y el órgano de gestión y administración que

\footnotetext{
${ }^{30}$ Art. 21 de la LC.
} 
ha de quedar concretado en los Estatutos sociales (Consejo Rector o el Administrador único), teniendo cada uno de ellos delimitado su ámbito de competencia. Junto a los mencionados, se incluyen los Interventores que integran el órgano de fiscalización de las cuentas anuales y del informe de gestión, salvo el supuesto en el que la sociedad cooperativa esté sometida a la auditoría externa ${ }^{31}$.

Además, las sociedades cooperativas pueden contar con otros órganos que son de carácter potestativo o no necesarios. Éstos son aquéllos que se van a establecer en razón de lo que determinen los Estatutos sociales, pero sin que su previsión limite las competencias de los órganos necesarios, como lo puede ser el Comité de Recursos que tramita y resuelve los recursos que se presenten contra las sanciones impuestas a los socios por el Consejo Rector $\mathrm{y}$ otras instancias de consulta o asesoramiento.

Centrándonos en la administración de la entidad cooperativa, ésta puede encomendarse a un órgano unipersonal (Administrador único) en los casos en los que la cooperativa cuente con un número de socios inferior a diez (nueve o menos miembros) (2) $^{32}$, lo habitual, que es la designación de un órgano colegiado (Consejo Rector). El Consejo Rector va a ser el órgano pluripersonal encargado de la gestión de la entidad ${ }^{33}$, siendo sus competencias básicas: la función social de gobierno de la cooperativa, la alta gestión de la entidad, la supervisión de los directivos y la representación de la cooperativa en relación con cualquier acto propio de las actividades que integren el objeto social. Junto a las indicadas, el Consejo Rector va a asumir de modo residual otras facultades que no se hubieran asignado a los órganos sociales por disposición normativa o estatutaria, siendo preciso en estos casos el previo acuerdo de la Asamblea general ${ }^{34}$. Las competencias indicadas han de ejercerse atendiendo al cumplimiento del objeto social de la cooperativa y de acuerdo con las exigencias de la Ley, los Estatutos sociales y la política prevista por la Asamblea general de socios.

${ }^{31}$ Arts. 19 y 62 de la LC.

${ }^{32}$ Autores como F.J. Alonso Espinosa, Órgano de administración, en: F.L. Alonso Espinosa (coord.), La sociedad cooperativa en la Ley 27/1999, de 16 de julio, de Cooperativas, Granada 2001, p. 231; F. Vicent Chuliá, El futuro de la legislación cooperativa, "Revista Jurídica de Economía Social y Cooperativa" 2002, No. 13, p. 40, manifiestan opiniones negativas en relación con la figura del Administrador único en el ámbito cooperativo.

${ }^{33}$ Art. 32 de la LC. Véanse J.I. Peinado Gracia, Capitulo I..., pp. 474-476; T. Vázquez Ruano, Progresiva aproximación del régimen de órgano de administración de las sociedades cooperativas al de las sociedades de capital, en: Mª.B. González Fernández, A. Cohen Benchetrit (dirs.), Derecho de sociedades. Revisando el derecho de sociedades de capital, Valencia 2018. A. Tato Plaza, La Administración, en: J.I. Peinado Gracia (dir.), T. Vázquez Ruano (coord.), Tratado de Sociedades Cooperativas, Valencia 2013, pp. 437-440.

${ }^{34}$ Art. $32.3^{\circ}$ de la LC. 
La elección de los consejeros de entre los socios de la cooperativa corresponde a la Asamblea que los determinará por mayoría de votos y mediante votación secreta. El período de este nombramiento será de entre tres y seis años, pudiendo ser reelegidos Los consejeros que hubieran agotado el plazo para el cual fueron elegidos continuará ostentando el cargo hasta el momento en el que produzca la aceptación de los que les sustituyan ${ }^{35}$ debido a que el simple nombramiento no es un acto completo ${ }^{36}$, pues dicha elección sólo surtirá efectos a partir de que el cargo sea aceptado por el socio. Y, necesariamente, ha de inscribirse en el Registro de Sociedades Cooperativas en el plazo de un mes $^{37}$.

El Consejo Rector estará compuesto por el número de miembros y cargos que se hubieran indicado estatutariamente, pero la norma determina que como mínimo se conforme de tres consejeros (personas físicas o jurídicas ${ }^{38}$ ) que son los que van a administrar la cooperativa (Presidente, Vicepresidente y Secretario $)^{39}$ y no pudiendo superar los quince, salvo que en la cooperativa sólo existan tres socios y se configurará por dos miembros (Presidente y Secretario, prescindiéndose del Vicepresidente). Los cargos mencionados serán elegidos, de entre los que formen parte del Consejo Rector, por el propio órgano o por la Asamblea general. Ello supone, en ocasiones, un impedimento para el adecuado funcionamiento de la sociedad. Razón por la que se entiende que la norma reconozca la posibilidad de que el Consejo confiera apoderamientos voluntarios (y también revocarlos) a cualquier persona y otorgue poderes especiales a cargos como el Gerente, el Director general o equivalentes. La concesión, modificación o revocación de los poderes de gestión o dirección permanentes se inscribirá en el Registro de Sociedades Cooperativas. Además de esta composición, estatutariamente podrá recogerse la existencia de otros cargos y de miembros suplentes, pudiendo nombrarse vocales o consejeros del Consejo Rector. El socio que sea designado miembro del Consejo Rector no puede hallarse inmerso en ningún supuesto de incompatibilidad, incapacidad o prohibición de los previstos $^{40}$. En el supuesto contrario, será destituido de manera inmediata a petición de cualquier socio, sin perjuicio de la responsabilidad en que pueda incurrir por llevar a cabo esta conducta desleal.

\footnotetext{
35 Art. 35 de la LC.

${ }^{36}$ Para más información: F.J. Alonso Espinosa, Órgano de administración, p. 233.

${ }^{37}$ Art. 34 de la LC.

${ }^{38}$ En el caso de ser persona jurídica se deberá designar a una persona física para el ejercicio de las funciones propias del cargo.

39 Art. 33 de la LC.

${ }^{40}$ Art. 41 de la LC. Para más información: A. Tato Plaza, La Administración, pp. 443-444.
} 
No obstante lo anterior, el adecuado desarrollo de las facultades que tiene atribuidas el órgano de gestión y administración de la cooperativa hace que sea posible que puedan ser nombrados consejeros personas que no ostenten la condición de socios, aunque con ciertas limitaciones. Así, se impone la precisión de que se trate de personas cualificadas y expertas, limitándose cuantitativamente su presencia (su número no puede exceder de un tercio del total); y, desde la perspectiva cualitativa, no podrán ser designados Presidente ni Vicepresidente.

El régimen de los administradores y su funcionamiento se determinará en los Estatutos sociales y, en defecto de dicha previsión, será la Asamblea general la que lo establezca. Del mismo modo, compete a la Asamblea la destitución de los administradores en cualquier momento y éstos también disponen de la facultad de renunciar al cargo cuando lo estimen, en cuyo caso su renuncia ha de aceptarla el Consejo Rector o la Asamblea general. Pero habrán de mantenerse en el cargo hasta tanto no se nombren sustitutos ${ }^{41}$.

En cuanto al funcionamiento del órgano de administración hay que añadir que los acuerdos del Consejo se adoptan por más de la mitad de los votos válidamente expresados, teniendo cada consejero un voto (el voto del Presidente salva los empates) y se levantará acta de las reuniones celebradas. Cabe la impugnación de los acuerdos adoptados que sean nulos o anulables en el plazo de dos meses o un mes, respectivamente ${ }^{42}$.

Por último, resulta relevante el régimen de responsabilidad de los miembros que forman parte del Consejo Rector recogido en la norma nacional. Esencialmente porque ésta hace una remisión expresa y genérica a la responsabilidad de los administradores de las sociedades anónimas ${ }^{43}$. En este sentido, y a pesar de que el legislador ha querido equiparar ambas formas societarias, el contenido previsto no determina si sólo se han de tener en cuenta las normas relativas a la acción social de responsabilidad o también las correspondientes a la acción individual y tampoco aspectos relacionados con el ejercicio de ambas acciones y la legitimación activa en cada caso. En igual sentido, se plantean dudas acerca del deber de diligencia exigida a los administradores de las sociedades cooperativas y sobre la extensión de responsabilidad en relación con supuestos concretos ${ }^{44}$.

${ }^{41}$ Art. 36 de la LC.

${ }^{42}$ Art. 37 de la LC.

${ }^{43}$ Art. 43 de la LC. Circunstancia que no ha sido bien recibida por la doctrina, véase A. Tato Plaza, La Administración, p. 451.

${ }^{44} \mathrm{Vid} . \mathrm{M}^{\mathrm{a}}$.J. Morillas Jarillo, M.I. Feliú Rey (dirs.), Curso de cooperativas, Madrid 2002, pp. 329-339, en cuanto a no promover la disolución de la entidad cuando sea preciso; A.J. Sequeira Martín, F. Sacristán Bergia, Una reflexión sobre la responsabilidad de los miembros del Consejo 


\section{Asociacionismo cooperativo en el marco agrario}

La integración cooperativa representativa hace referencia a los supuestos en los que la relación entre las sociedades cooperativas no se singulariza por tener una finalidad económica de manera exclusiva. Antes bien, la primacía se otorga al interés general o a la representación pública de un sector ${ }^{45}$. El principio reconocido por la ACI de la cooperación intercooperativa trae como consecuencia que se produzca una estructuración de plataformas de colaboración de carácter libre y voluntario. De este modo, es posible tanto la integración cooperativa como otras formas de unión de cooperativas de diferentes peculiaridades. Distinguiéndose el asociacionismo cooperativo que tienen por finalidad la defensa y representación de los intereses generales de las cooperativas y del cooperativismo no empresarial (la intercooperación representativa); de la colaboración entre sociedades cooperativas con la pretensión de fomentar e impulsar su actuación empresarial, la cual se corresponde con la intercooperación económica. En el ámbito agrario, el asociacionismo ha de entenderse como una forma de organización de los esfuerzos de los participantes para conseguir un incremento de la valoración de la producción y una tutela única de los intereses de los mismos.

En consonancia con ello, y junto a las cooperativas de segundo (o ulterior) grado y de los grupos cooperativos, la norma nacional prevé que las cooperativas de cualquier tipo y clase podrán constituir sociedades, agrupaciones, consorcios y uniones entre sí, o con otras personas físicas o jurídicas, públicas o privadas, $\mathrm{y}$ formalizar convenios o acuerdos para cumplir su objeto social de manera adecuada y obtener una mejor protección de sus intereses ${ }^{46}$. Las clases de asociacionismo cooperativo van a ser las siguientes. De un lado, las Uniones conformadas por tres cooperativas de la misma clase como mínimo y que podrán integrarse en otra unión existente o constituir una nueva. De otro, las Federaciones que se conforman por cooperativas o por uniones de cooperativas o por ambas formas, precisándose que-como mínimo-asocien diez cooperativas que no sean todas de la misma clase. Y, por último, las

Rector de las Cooperativas, "Revista Derecho de Sociedades" 2003, No. 21, pp. 219-232; A. Tato Plaza, La Administración, pp. 455-459.

45 J.M. Embid Irujo, Problemas actuales de la integración cooperativa, "Revista de Derecho Mercantil" 1998, No. 227, pp. 7-36.

${ }^{46}$ Art. 79 y arts. 118 a 120 de la LC. Para más información véase, E. Dávila Millán, Capitulo XII. Asociacionismo y representación del movimiento cooperativo, en: J.I. Peinado Gracia (dir.), T. Vázquez Ruano (coord.), Tratado de Derecho..., pp. 1275-1288; J.M. Embid Irujo, R. Alfonso Sánchez, Capitulo XI..., pp. 1265-1270. 
Confederaciones que surgen por la asociación de uniones de cooperativas y las federaciones. En cuyo caso es necesario, al menos, tres federaciones de cooperativas que agrupen a cooperativas de tres Comunidades autónomas diversas.

Cualquiera de estas entidades adquiere personalidad jurídica una vez que la escritura pública de constitución se inscriba en el Registro de Sociedades Cooperativas, la cual hará referencia no sólo a las entidades promotoras, al acuerdo de constitución y a los Estatutos sociales, sino también a los que van a integrar los órganos de representación y gobierno.

La finalidad esencial que distingue a estas entidades es la representación y defensa de los intereses generales de las sociedades cooperativas y de sus socios ante las Administraciones Públicas y ante las personas físicas o jurídicas. Además de lo señalado, se le reconocen otras funciones concretas, cuáles son: el fomento de la promoción y formación cooperativa; el ejercicio de la conciliación en los conflictos surgidos entre las cooperativas que asocien o entre éstas y sus socios; la organización de servicios de asesoramiento, auditorías, asistencia jurídica o técnica en interés de sus socios; y ser interlocutores y representantes ante las entidades y los organismos públicos.

La norma que se ocupa de la regulación de la integración cooperativa en el ordenamiento jurídico nacional es la Ley $13 / 2013^{47}$, la cual se encarga no sólo del fomento de la integración de cooperativas, sino también de otras entidades asociativas de carácter agroalimentario. El objetivo fundamental de este texto normativo se centra en el impulso de la fusión e integración de las cooperativas agrarias y del resto de formas asociativas agroalimentarias, entre las que se incluyen los ya referidos grupos cooperativos que asocian a varias cooperativas con la entidad cabeza de grupo y que ejercitan facultades u otorgan instrucciones de obligado cumplimiento para sus integrantes. Produciéndose una unidad de decisión respecto de esas facultades. Además, el legislador trata de promover la creación de grupos cooperativos y de agrupaciones de entidades asociativas con el fin de que lleguen a ser una Entidad Asociativa Agroalimentaria de carácter Prioritario. Ha de tenerse en cuenta que van a ser entidades asociativas que conforman el primer eslabón de la cadena alimentaria, las siguientes: las sociedades cooperativas, las coopera-

${ }^{47}$ Ley 13/2013, de 2 de agosto, de fomento de la integración de cooperativas y de otras entidades asociativas de carácter agroalimentario (BOE No. 185, de 6 de abril). Sobre dicha norma puede consultarse: E. Meliá Martí, M. Peris Mendoza, Los procesos de integración de las cooperativas agroalimentarias. De la norma a la realidad. Especial referencia a la Ley 13/2013 de Fomento de la Integración Cooperativa, "Revista de Estudios Cooperativos" 2017, No. 126, pp. 177-197. 
tivas de segundo grado, los grupos cooperativos, las sociedades agrarias de transformación, las organizaciones de productores con personalidad jurídica propia, y las entidades civiles o mercantiles. Estas últimas, siempre que más del $50 \%$ de su capital social pertenezca a cooperativas, a organizaciones de productores o a sociedades agrarias de transformación.

La calificación como Entidad Asociativa Agroalimentaria Prioritaria precisa del cumplimiento de una serie de requisitos que el propio legislador se ha encargado de enumerar, a saber: que se trate de una entidad asociativa agroalimentaria de las reconocidas en la norma; que su implantación y ámbito de actuación económico sea supra-autonómico; que comercialicen de forma conjunta la totalidad de la producción de las entidades asociativas y de los productores que las componen; que la facturación de la entidad solicitante, o la suma de las facturaciones de las que se fusionan o integren, alcance la cantidad mínima precisada en la norma reglamentaria y que dependerá de los sectores productivos (según la Clasificación Nacional de Actividades Económicas); y que conste expresamente en los Estatutos o en las disposiciones reguladoras correspondientes, la obligación de los productores de entregar la totalidad de su producción para la comercialización en común; al igual que contemplen las necesarias previsiones para garantizar a sus productores asociados el control democrático de su funcionamiento y de la adopción de sus decisiones. Evitándose, de este modo, la posición de dominio de uno o varios de sus miembros.

El cumplimiento de los presupuestos señalados, permite que el Ministerio de Agricultura, Alimentación y Medio Ambiente pueda reconocer la Entidad Asociativa Prioritaria, siguiendo el procedimiento reglamentario establecido a tal fin, y la inscripción en el correspondiente Registro Nacional de Entidades Asociativas Prioritarias. En concreto, se hace referencia al proceso determinado en el Real Decreto 550/2014, por el que se desarrollan los requisitos y el procedimiento para el reconocimiento de las Entidades Asociativas Prioritarias y para su inscripción y baja en el Registro Nacional de Entidades Asociativas Prioritarias ${ }^{48}$, el cual ha sido modificado recientemente por el Real Decreto 161/2019, de 22 de marzo $^{49}$ en sus aspectos técnicos. Esto es,

${ }^{48}$ Real Decreto 550/2014, de 27 de junio, por el que se desarrollan los requisitos y el procedimiento para el reconocimiento de las Entidades Asociativas Prioritarias y para su inscripción y baja en el Registro Nacional de Entidades Asociativas Prioritarias, previsto en la Ley 13/2013, de 2 de agosto, de fomento de la integración de cooperativas y de otras entidades asociativas de carácter agroalimentario (BOE No. 173, de 17 de julio). En particular, arts. 3 a 6 del citado texto normativo.

49 Real Decreto 161/2019, de 22 de marzo, por el que se modifica el Real Decreto 550/2014, de 27 de junio, por el que se desarrollan los requisitos y el procedimiento para el reconocimiento de las Entidades Asociativas Prioritarias y para su inscripción y baja en el Registro Nacional de 
para mejorar la eficacia de las Entidades Asociativas Prioritarias y cumplir los fines de la política agroalimentaria y, al mismo tiempo, la gestión de las mismas y su eficiencia en el mercado. El fortalecimiento de las estructuras asociativas y el incremento de su dimensión facilitarán el desarrollo de la innovación y la incorporación de nuevas tecnologías, aumentarán su productividad y eficiencia y mejorarán su capacidad de competir más eficazmente, tanto en el mercado nacional como en los mercados internacionales, pudiendo hacer frente a los retos de una economía global en las mejores condiciones en cuanto a capacidad de negociación.

\section{BIBLIOGRAFÍA}

Alfonso Sánchez R. (2000), La integración cooperativa y sus técnicas de realización: la cooperativa de segundo grado, Valencia.

Alonso Espinosa F.J. (2001), Órgano de administración, en: F.J. Alonso Espinosa (coord.), La sociedad cooperativa en la Ley 27/1999, de 16 de julio, de Cooperativas, Granada.

Dávila Millán E., (2019), Capítulo XII. Asociacionismo y representación del movimiento cooperativo, en: J.I. Peinado Gracia (dir), T. Vázquez Ruano (coord.), Tratado de Derecho de sociedades cooperativas, vol. II, Valencia.

Embid Irujo J.M. (1998), Problemas actuales de la integración cooperativa, "Revista de Derecho Mercantil" No. 227.

Embid Irujo J.M. (2019), Derecho de sociedades y economía social: nuevos retos, en: A. Emparanza Sobejano (dir.), El Derecho de sociedades y de cooperativas: nuevos retos en su configuración y en la gestión de los administradores, Madrid.

Embid Irujo J.M., Alfonso Sánchez R. (2019), Capitulo XI. III. Formas no personificadas de integración: grupos cooperativos, en: J.I. Peinado Gracia (dir.), T. Vázquez Ruano (coord.), Tratado de Derecho de Sociedades Cooperativas, vol. II, Valencia.

González Fernández Mª.B., Cohen Benchetrit A. (dirs.) (2018), Derecho de sociedades. Revisando el derecho de sociedades de capital, Valencia.

Meliá Martí E., Peris Mendoza M. (2017), Los procesos de integración de las cooperativas agroalimentarias. De la norma a la realidad. Especial referencia a la Ley 13/2013, de Fomento de Integración Cooperativa, "Revista de Estudios Cooperativos" No. 126.

Morillas Jarillo Ma.J. (2019), Capitulo II. Concepto y clases de cooperativas, en: J.I. Peinado Gracia (dir.), T. Vázquez Ruano (coord.), Tratado de Derecho de sociedades cooperativas, vol. II, Valencia.

Morillas Jarillo Ma.J., Feliú Rey M.I. (2002), Curso de cooperativas, Madrid.

Peinado Gracia J.I. (2019), Capitulo I. Normas y ámbito de aplicación, en: J.I. Peinado Gracia (dir.), T. Vázquez Ruano (coord.), Tratado de Derecho de sociedades cooperativas, vol. II, Valencia.

Entidades Asociativas Prioritarias, previsto en la Ley 13/2013, de 2 de agosto, de fomento de la integración de cooperativas y de otras entidades asociativas de carácter agroalimentario (BOE No. 83, de 6 de abril). 
Peinado Gracia J.L. (dir.), Vázquez Ruano T. (coord.) (2013), Tratado de sociedades cooperativas, Valencia.

Sequeira Martín A.J., Sacristán Bergia F. (2003), Una reflexión sobre la responsabilidad de los miembros del Consejo Rector de las Cooperativas, "Revista Derecho de Sociedades" No. 21.

Server Izquierdo R., Meliá Martí E. (1999), Caracterización empresarial de los grupos y otras formas de integración cooperativa al amparo del nuevo marco legislativo, "Revista de Estudios Cooperativos" No. 69.

Tato Plaza A., (2013), La Administración, en: J.I. Peinado Gracia (dir.), T. Vázquez Ruano (coord.), Tratado de Sociedades Cooperativas, Valencia.

Vargas Vasserot C. (2010), Integración y diferenciación cooperativa: de las secciones a los grupos de sociedades, "Boletín de la Asociación Internacional de Derecho Cooperativo" No. 44.

Vargas Vasserot C., Gadea Soler E., Sacristán Bergia F. (2017), Régimen económico, integración, modificaciones estructurales y disolución, Madrid.

Vázquez Pena J.M. (2019), Capitulo XI. II. Formas personificadas de integración: cooperativas de segundo grado, en: J.I. Peinado Gracia (dir.), T. Vázquez Ruano (coord.), Tratado de Derecho de sociedades cooperativas, vol. II, Valencia.

Vázquez Ruano T. (2018), Progresiva aproximación del régimen de órgano de administración de las sociedades cooperativas al de las sociedades de capital, en: Mª. B. González Fernández, A. Cohen Benchetrit (dirs.), Derecho de sociedades. Revisando el Derecho de sociedades de capital, Valencia.

Vicent Chuliá F. (2019), Capítulo I. Introducción, en: J.I. Peinado Gracia (dir.), T. Vázquez Ruano (coord.), Tratado de Derecho de sociedades cooperativas, vol. II, Valencia.

Vicent Chuliá F. (2002), El futuro de la legislación cooperativa, "Revista Jurídica de Economía Social y Cooperativa" No. 13.

\title{
COOPERATIVES IN THE AGRI-FOOD ENVIRONMENT FROM A PRIVATE LAW PERSPECTIVE
}

\author{
Summary
}

Organisations made up of individuals who join together to achieve a common goal by taking joint action are a preferred form of activity in the agricultural sector and refers to cooperative societies and the advantages they provide from the point of view of the individual small grower or breeder. The general legal regime of cooperatives was established in Spain by Act 27/1999 of 16 July 1999, but there are also a number of autonomous regulations that also govern this sector. The considerations presented in the article, thanks to the analysis of important practical aspects such as the observance of the guiding principles, as well as the structure and operation of cooperatives, show their practical importance in the agricultural sector in Spain.

Keywords: cooperatives, agri-food environment, agricultural sector 


\title{
UNA NOTA DI DIRITTO PRIVATO SULLE COOPERATIVE NELL'AMBIENTE AGROALIMENTARE
}

\begin{abstract}
Riassunto
Le entità composte da persone che si uniscono per raggiungere un obiettivo comune realizzando attività congiunte sono una forma favorevole di business nel settore agricolo. Ci riferiamo alle società cooperative e ai vantaggi che portano dal punto di vista del singolo piccolo agricoltore o allevatore. La legge spagnola che stabilisce il regime giuridico generale delle cooperative è la legge 27/1999 del 16 luglio 1999. Tuttavia, ci sono anche vari regolamenti autonomi approvati in questo settore. Lo studio qui presentato evidenzia la rilevanza pratica delle cooperative nel settore agricolo in Spagna, analizzando aspetti fondamentali che si presentano nella pratica, come il rispetto dei principi guida e la struttura, il funzionamento di questa entità.
\end{abstract}

Parole chiave: cooperative, ambiente agroalimentare, settore agricolo 